\title{
Her-2 expression in feline mammary lesions
}

\author{
Lígia Fernandes Gundim ${ }^{1}$, Taís Meziara Wilson², Nicolle Pereira Soares ${ }^{1}$, \\ Igor de Paula Castro ${ }^{1}$, Mariana Ribeiro Castro ${ }^{1}$, Alessandra Aparecida Medeiros-Ronchi ${ }^{1}$ \\ ${ }^{1}$ Federal University of Uberlândia, Animal Pathology Laboratory, Uberlândia, MG, Brazil \\ ${ }^{2}$ University of Brasília, Animal Pathology Laboratory, Brasília, Brazil
}

Received May 31, 2018

Accepted February 12, 2019

\begin{abstract}
Due to the high occurrence of mammary neoplasms in cats, the search for prognostic factors has recently intensified. Human epidermal growth factor receptor type 2 (Her-2) is prominent among the immunohistochemical markers used for prognosis. The aim of this study was to evaluate the expression of Her-2 in mammary lesions in queens and relate the expression to the type and histological grade of mammary carcinomas. Thirty-eight cases were selected from 2006 to 2016 and were classified and histologically graded. Her-2 expression was determined through immunohistochemistry, using a semi-quantitative analysis of the slides according to the scores recommended in the Dako HercepTest. Chi-square test was used to evaluate the relationship between Her-2 expression and mammary lesion type and the type and histological grade of carcinoma $(P<0.05)$. The mean age was 8.5 years and young cats were affected only by benign hyperplasias and neoplasms. Undefined cats were more affected $(84.37 \%)$, followed by Siamese $(9.37 \%)$ and Persian (6.25\%) cats. Regarding the histological classification, $18.42 \%$ of the mammary lesions were classified as hyperplasia, $18.42 \%$ as adenoma, and $63.16 \%$ as carcinoma. The most common type of carcinoma was tubulopapillary carcinoma (66.7\%), followed by solid carcinoma (25\%) and cribriform carcinoma (8.33\%). Her-2 overexpression was observed in four samples (16.7\%) of tubulopapillary carcinoma, two grade I carcinomas and two grade II carcinomas, but no relationship was observed between Her-2 expression and type of mammary lesion $(P=0.3127)$, carcinoma type $(0.3446)$, or histological grade $(0.6485)$. In conclusion, Her-2 overexpression is unrelated to histological grade and type.
\end{abstract}

c-erbB-2/neu, feline, imunohistochemistry, mammary tumour

Mammary neoplasms are the third most frequent tumour type in felines and most frequently affect females, adults, and uncastrated queens, with no racial predisposition (Lana et al. 2007). Several prognostic factors have already been established for these tumours, such as clinical stage, tumour size (Ito et al. 1996), histological grade (Castagnaro et al. 1998, Hughes and Dobson 2012, De Campos et al. 2015), mitosis index (Preziosi et al. 2002, Hughes and Dobson 2012), and lymphovascular invasion (Seixas et al. 2011). Moreover, in recent years, immunohistochemical markers have been used to obtain a clearer understanding of the molecular pathogenesis in feline neoplasms (Hughes and Dobson 2012).

Mammary carcinomas in cats have histological features (Pérez-Alenza et al. 2004) and metastatic (Macewen 1990) and hormonal dependence (De Las Mulas et al. 2000) similar to human mammary neoplasms, indicating that results from animal models can be extrapolated to female humans (De Maria et al. 2005).

Studies have indicated that human epidermal growth factor receptor type 2 (Her-2) expression in cats is associated with a larger tumour size, higher histological grade (Ordás et al. 2007), and lower survival rate (Ordás et al. 2007, De Campos et al. 2015). However, some authors have stated that, in contrast to women, Her-2 may not be involved in the carcinogenesis of feline mammary tumours and its value as a prognostic factor in queens has 
been questioned because hyperplasias and malignant and benign tumours both overexpress Her-2 (Rasotto et al. 2011).

Studies addressing Her-2 expression in queens are scarce and, therefore, research is necessary to elucidate its role as a prognostic factor. The aim of this study was to evaluate Her-2 immunoexpression in hyperplasias and benign and malignant neoplasms in female cats, and to relate this immunoexpression to histological type and grade, which are crucial histological indicators of mammary carcinomas.

\section{Materials and Methods}

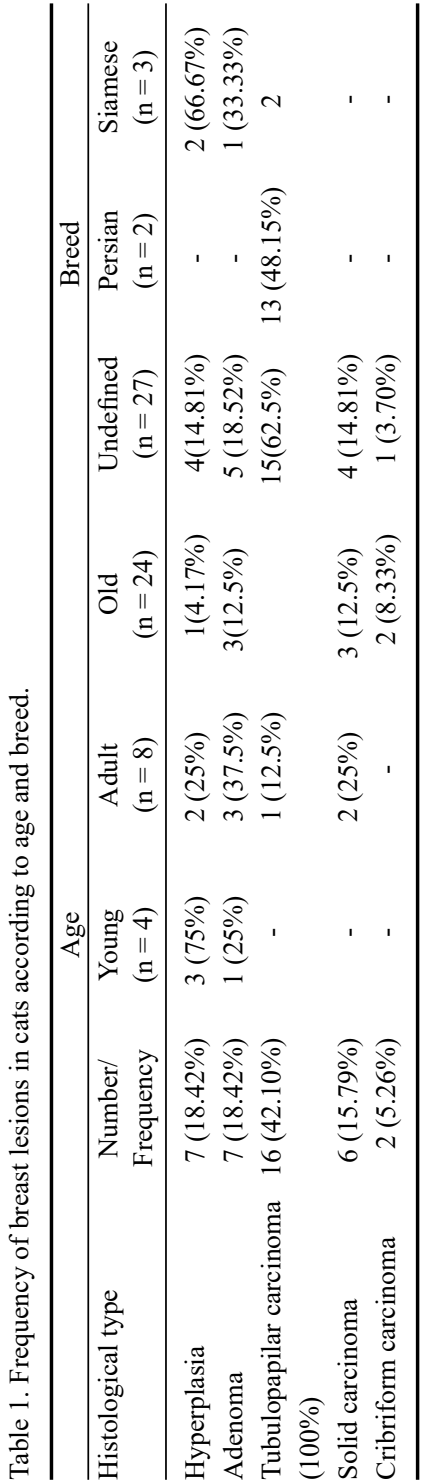

A retrospective study was conducted between 2006 and 2016, examining mammary neoplasms (benign or malignant) and nonneoplastic lesions in female cats. Epidemiological data (sex, breed, animal age, and lesion size) were collected. Tumour size was grouped according to the recommendations of McNeill et al. (2009) and age was grouped according to the recommendations of Togni et al. (2013).

Histological slides with mammary lesions were revised by two pathologists, classified according to the method of Misdorp et al. (1999), and histologically graded according to the method of Elston and Ellis (1993).

Immunohistochemistry was performed using the streptavidinbiotin-peroxidase technique. Antigenic recovery was induced by microwave heating (ethylenediaminetetraacetic acid buffer, $\mathrm{pH} 9.0$, $20 \mathrm{~min}$ ). Subsequently, endogenous peroxidase was blocked with a $10 \%$ hydrogen peroxide solution. The first antibody (Her-2 Dako Cytomation polyclonal antibody A0485) was diluted in bovine serum albumin (BSA) at 1:400 and was incubated in a moist chamber overnight. This was followed by incubation for 30 min with a secondary antibody and with the avidin-biotin-peroxidase complex (Dako) for a further $30 \mathrm{~min}$ and revelation was performed with diaminobenzene (Dako K3468-1) for $3 \mathrm{~min}$. Mammary carcinoma was used as a positive control and in the negative control, the primary antibody was replaced with a diluent (BSA).

Her-2 overexpression was determined through semiquantitative analysis of the membrane marking of the epithelial cells, according to the scores recommended in the HercepTest (Dako).

The relationship between Her-2 expression and type of mammary lesion (hyperplasia, adenoma, and carcinoma) and histological type (tubulopapillary, solid, cribriform) and histological grade of carcinoma was determined using chi-square test $\left(\chi^{2}\right) 2.5$ in Microsoft Excel 2007 $(P<0.05)$.

\section{Results}

Thirty-eight feline health records were selected, two samples were excluded due to a lack of data on age, and six for a lack of data on breed. The mean age was 8.5 years (range 6 months to 15 years), with four young animals $(11.11 \%)$, eight adults $(22.22 \%)$, and 24 older animals $(66.67 \%)$. The young cats were affected only by hyperplasias $(3 / 4 ; 75 \%)$ and benign tumours $(1 / 4 ; 25 \%)$, as were most adult cats $(5 / 8,62.5 \%)$. In contrast, older cats were affected mostly by carcinomas $(20 / 24,83.33 \%)$ (Table 1).

In terms of breed, mammary lesions occurred more frequently in undefined cats $(84.37 \%)$, followed by Siamese $(9.37 \%)$, and Persian $(6.25 \%)$ cats. Of the 
38 breast lesions, $24(63.16 \%)$ were malignant tumours, among which tubulopapillary carcinoma was the most frequent type (66.67\%) (Table 1$)$.

Data on tumour size were available in 11 health records carcinoma, with three tumours classified as T1 (27.27\%), four as T2 (36.36\%), and four as T3 (36.36\%). Regarding location, three $(10 \%)$ tumours were found in the cranial thoracic gland, two $(6.67 \%)$ were in the caudal thoracic gland, six (20\%) in the cranial abdominal gland, eight $(26.67 \%)$ in the caudal abdominal gland, and $11(36.67 \%)$ in multiple breasts. In eight instances, these data were unavailable.

Only carcinomas were graded (24 samples). Ten carcinomas were grade I $(41.67 \%)$, all of which were tubulopapillary carcinomas; ten were grade II (41.67\%), of which six were tubulopapillary carcinomas $(60 \%)$, three were solid carcinomas $(30 \%)$, and one was cribriform carcinoma (10\%). Only four carcinomas were grade III $(16.67 \%)$, of which three were solid carcinomas $(75 \%)$ and one was cribriform carcinoma (25\%).

Regarding Her-2 expression, of the 24 carcinoma samples, four (16.7\%) were positive, of which one received a score of $3+$ and three received a score of $2+$ (Plate II, Fig. 1). All carcinomas overexpressing Her-2 were tubulopapillary, with two being grade I (50\%) and two being grade II (50\%). Membrane staining occurred only in the tumour epithelial cells, with no staining in normal epithelial cells; in some cases, weak cytoplasmic marking was observed. No sample with benign neoplasia or hyperplasia exhibited membrane staining for Her-2.

No correlation was observed between immunolabelling and the mammary lesion type (carcinomas, adenomas, or hyperplasias) $(P=0.3127)$, carcinoma histological type (tubulopapillary, solid, or cribriform) $(P=0.3467)$, or histological grade $(P=0.6485)$.

\section{Discussion}

Young cats were more affected by hyperplasia (75\%), which may be associated with hyperplasia fibroepithelial aetiology, which has been linked to the natural progestogens produced from the first heat (Hayden et al. 1981). The mean age of cats affected by carcinomas (10.31 years) is in line with other studies, which have described the age as varying between 10 and 12 years (Millanta et al. 2006; Lana et al. 2007; Kustritz 2007; Cunha et al. 2016). In older cats, the occurrence of carcinomas is higher than that of benign tumours (Hayes et al. 1985) and, although evidence indicates that prognosis is worse in older cats, age has no significant association with post-surgical survival (Castagnaro et al. 1998).

The higher occurrence of mammary alterations in non-pedigree cats may be associated with the population stratum presenting at the Veterinary Hospital of the Federal University of Uberlândia and breed differences among the populations studied makes it difficult to establish this relationship. Studies conducted in Brazil by Togni et al. (2013) and Cunha et al. (2016) revealed a higher occurrence in non-pedigree cats.

Regarding the size of the tumour masses, the majority $(72.72 \%)$ were larger than $2 \mathrm{~cm}$ in diameter. Togni et al. (2013) and De Campos et al. (2015) reported that tumours smaller than $2 \mathrm{~cm}$ were the most common in cats. Tumour size is a critical prognostic factor because larger tumours have a greater number of cell divisions and a higher probability of progression to malignant behaviour as a result of the accumulation of mutations (Matos et al. 2012). Furthermore, cats with larger tumour masses are known to have lower survival (Ito et al. 1996).

Regarding location, the abdominal breasts were more affected, as reported by De Campos et al. (2015). Some authors have divided the mammary gland into thoracic, abdominal, and inguinal areas, as recommended by Dyce et al. (2004), reporting a higher occurrence of tumours in the abdominal (Shafiee et al. 2013) and inguinal glands (Cunha et al. 2016). 
Malignant tumours were more frequent than benign lesions, and tubulopapillary carcinoma was the most frequent. In Italian studies, Rasotto et al. (2011) considered only neoplasms and reported that $88 \%$ of tumours were classified as carcinomas, whereas Maniscalco et al. (2012) also considered hyperplasias as benign lesions and found that $69.2 \%$ were malignant.

Regarding carcinoma classification, most authors have reported tubulopapillary carcinoma as the most frequent (Togni et al. 2013), followed by solid carcinomas (Pereira et al. 2004; Millanta et al. 2006; Maniscalco et al. 2012; De Campos et al. 2015) as in this study. However, some studies have not mentioned cribriform carcinoma (Pereira et al. 2004; Millanta et al. 2006) and other histological types, such as mucinous carcinoma, glycogen-rich carcinoma, and carcinosarcoma (De Campos et al. 2015). Tubulopapillary carcinomas express more Ki-67 than solid and cribriform carcinomas (Pereira et al. 2004), indicating a higher proliferative index. In addition, cats with cribriform carcinoma have the lowest survival, followed by those with solid carcinoma and tubulopapillary carcinoma (Seixas et al. 2011; Mills et al. 2015).

According to Hughes and Dobson (2012), histological classification of neoplasms should be accompanied by histological grading for a more reliable prognostic evaluation. In the present study, the majority of tumours $(84 \%)$ were grade I or II. Other authors have also reported that most feline carcinomas were well differentiated (grades I and II) (Silva 2016) or that carcinomas showed a similar distribution between grades (Rasotto et al. 2011; De Campos et al. 2015).

Previous studies have reported an overexpression of Her-2 in cats ranging from $12.3 \%$ to $40 \%$ (De Maria et al. 2005; Ordás et al. 2007; Rasotto et al. 2011; De Campos et al. 2015), and $16.7 \%$ of the carcinomas in this study presented overexpression. Variation in results may occur because of a lack of standardization of the immunohistochemical technique (Matos et al. 2012; De Campos et al. 2015) and the use of human antibodies may present low specificity (Matos et al. 2012). However, Soares et al. (2012) tested several protocols and antibodies for Her-2 expression verification and found that the clone used in the present study (A0485) obtained the best results.

In contrast to the present study, previous studies have reported an association of Her-2 overexpression with greater tumour size, higher histological grade, absence of hormone receptor expression (Ordás et al. 2007), and lower overall survival (De Campos et al. 2015).

In the present study, benign tumours and hyperplasias did not present overexpression of Her-2, as in a previous study conducted in Spain (Ordás 2007). Conversely, a study conducted in Italy described $6.8 \%$ of hyperplasias and $10 \%$ of adenomas as positive, suggesting that Her-2 overexpression may be associated with the growth of these benign lesions (Rasotto et al. 2011). However, a small number of benign samples may have impaired the expression in this study.

Despite Her-2 expression being a critical prognostic factor for women with breast cancer and the similarity between breast tumours in women and cats, the percentage of breast cancers expressing Her-2 in cats is lower than that in women. In addition, no correlation was observed between grade and histological type and Her-2 expression, indicating that in cats, Her-2 overexpression may be considered an independent prognostic factor. Benign breast lesions in cats apparently do not express Her-2.

\section{References}

Castagnaro M, Casalone C, Bozzetta E, De Maria R, Biolatti B, Caramelli M 1998: Tumour grading and the one-year post-surgical prognosis in feline mammary carcinomas. J Comp Path 119: 263-275

Cunha S, Corgozinho K, Justen H, Silva K, Leite J, Ferreira AM. 2016: Survival and disease-free interval of cats with mammary carcinoma treated with chain mastectomy. Acta Sci Vet 44: 1-8 
De Campos CB, Damasceno KA, Gamba CO, Ribeiro AM, Machado CJ, Lavalle GE, Cassali GD 2015: Evaluation of prognostic factors and survival rates in malignant feline mammary gland neoplasms. J Fel Med Surg 18: 1003-1012

De las Mulas JM, Van Niel M, Millan Y, Blankenstein MA, Van Mil F, Misdorp W 2000. Immunohistochemical analysis of estrogen receptors in feline mammary gland benign and malignant lesions: comparison with biochemical assay. Domest Anim Endocrinol 18: 111-125

De Maria R, Olivero M, Iussich S, Nakaichi M, Murata T, Biolatti B, Di Renzo MF 2005. Spontaneous feline mammary carcinoma is a model of HER2 overexpressing poor prognosis human breast cancer. Cancer Res $\mathbf{6 5}$ : 907-912

Dyce M, Sack W, Wensing C 2004. Glândula mamária. Tratado de Anatomia Veterinária. Rio de Janeiro: Elsevier, pp. $357-358$

Elston EW, Ellis IO 1993. Method for grading breast cancer. J Clin Path 46: 189

Hayes HM Jr., Milne KL, Mandell CP 1985. Epidemiological features of feline mammary carcinoma. Vet Rec 108: $476-479$

Hayden DW, Johnston SD, Kiang DT, Johnson KH, Barnes DM 1981. Feline mammary hypertrophy/fibroadenoma complex: clinical and hormonal aspects. A J Vet Res 42: 1699-1703

Hughes K, Dobson JM 2012. Prognostic histopathological and molecular markers in feline mammary neoplasia. Vet J 194: 19-26

Ito T, Kadosawa T, Mochizuki M, Matsunaga S, Nishimura R, Sasaki N 1996. Prognosis of malignant mammary tumor in 53 cats. J Vet Med Sci 58: 723-726

Kustritz MVR 2007. Determining the optimal age for gonadectomy of dogs and cats. JAVMA 231: 1665-1675

Lana SE, Rutteman GR, Withrow SJ 2007. Tumors of the mammary gland. In: Withrow SJ, Page R, Vail DM (Eds). Small Animal Clinical Oncology. Elsevier, pp. 619-636

McNeill CJ, Sorenmo KU, Shofer FS, Gibeon L, Durham AC, Barber LG, Overley B 2009. Evaluation of adjuvant doxorubicin-based chemotherapy for the treatment of feline mammary carcinoma. J Vet Intern Med 23: $123-129$

MacEwen EG 1990. Spontaneous tumors in dogs and cats: models for the study of cancer biology and treatment. Cancer Metastasis Rev 9: 125-136

Maniscalco L, Iussich S, de las Mulas JM, Millán Y, Biolatti B, Sasaki N, De Maria R 2012. Activation of AKT in feline mammary carcinoma: a new prognostic factor for feline mammary tumours. Vet J 191: 65-71

Matos AJF, Baptista CS, Gärtner MF, Rutteman GR 2012. Prognostic studies of canine and feline mammary tumours: the need for standardized procedures. Vet J 193: 24-31

Millanta F, Citi S, Della Santa D, Porciani M, Poli A 2006. COX-2 expression in canine and feline invasive mammary carcinomas: correlation with clinicopathological features and prognostic molecular markers. Breast Cancer Res Treat 98: 115-120

Mills SW, Musil KM, Davies JL, Hendrick S, Duncan C, Jackson ML, Simko E 2015. Prognostic value of histologic grading for feline mammary carcinoma a retrospective survival analysis. Vet Pathol 52: 238-249

Misdorp W (Ed.) 1999. Histological classification of mammary tumors of the dog and the cat. Armed Forces Institute of Pathology: American Registry of Pathology: World Health Organization Collaborating Center for Comparative Oncology.

Ordás J, Millán Y, Dios R, Reymundo C, de las Mulas JM 2007. Proto-oncogene HER-2 in normal, dysplastic and tumorous feline mammary glands: an immunohistochemical and chromogenic in situ hybridization study. BMC Cancer 7: 179

Pérez-Alenza MD, Jiménez Á, Nieto AI, Peña L 2004. First description of feline inflammatory mammary carcinoma: clinicopathological and immunohistochemical characteristics of three cases. Breast Cancer Res 6: R300

Preziosi R, Sarli G, Benazzi C, Mandrioli L, Marcato PS 2002. Multiparametric survival analysis of histological stage and proliferative activity in feline mammary carcinomas. Res Vet Sci 73: 53-60

Rasotto R, Caliari D, Castagnaro M., Zanetti R, Zappulli V 2011. An immunohistochemical study of HER-2 expression in feline mammary tumours. J Comp Pathol 144: 170-179

Seixas F, Palmeira C, Pires MA, Bento MJ, Lopes C 2011. Grade is an independent prognostic factor for feline mammary carcinomas: a clinicopathological and survival analysis. Vet J 187: 65-71

Shafiee R, Javanbakht J, Atyabi N, Bahrami A, Kheradmand D, Safaei R, Hosseini E 2013. Comparative value of clinical, cytological, and histopathological features in feline mammary gland tumors; an experimental model for the study of human breast cancer. Diagn Pathol 8: 136

Pereira PD, Carvalheira J, Gärtner F 2004. Cell proliferation in feline normal, hyperplastic and neoplastic mammary tissue-an immunohistochemical study. Vet J 168: 180-185

Silva MJCS 2016. Clinical and molecular characterization of feline mammary carcinomas overexpressing HER2 proto-oncogene (FMC-HER2+): new strategies for effective diagnostic and cancer therapy. Doctoral thesis. Universidade de Lisboa, Faculdade de Medicina Veterinária, Lisboa

Soares M, Correia J, Carvalho S, Ferreira F 2012. Evaluation of fHER2 expression in feline mammary tumours and its correlation with clinicopathological features (in Portuguese). RPCV 111: 191-198

Togni M, Masuda EK, Kommers GD, Fighera RA, Irigoyen LF 2013. A retrospective study of 207 cases of mammary tumours in queens (in Portuguese). Pesq Vet Bras 33: 353-358 
Plate II

Gundim L. F. et al.: Her-2 expression ... pp. 43-47
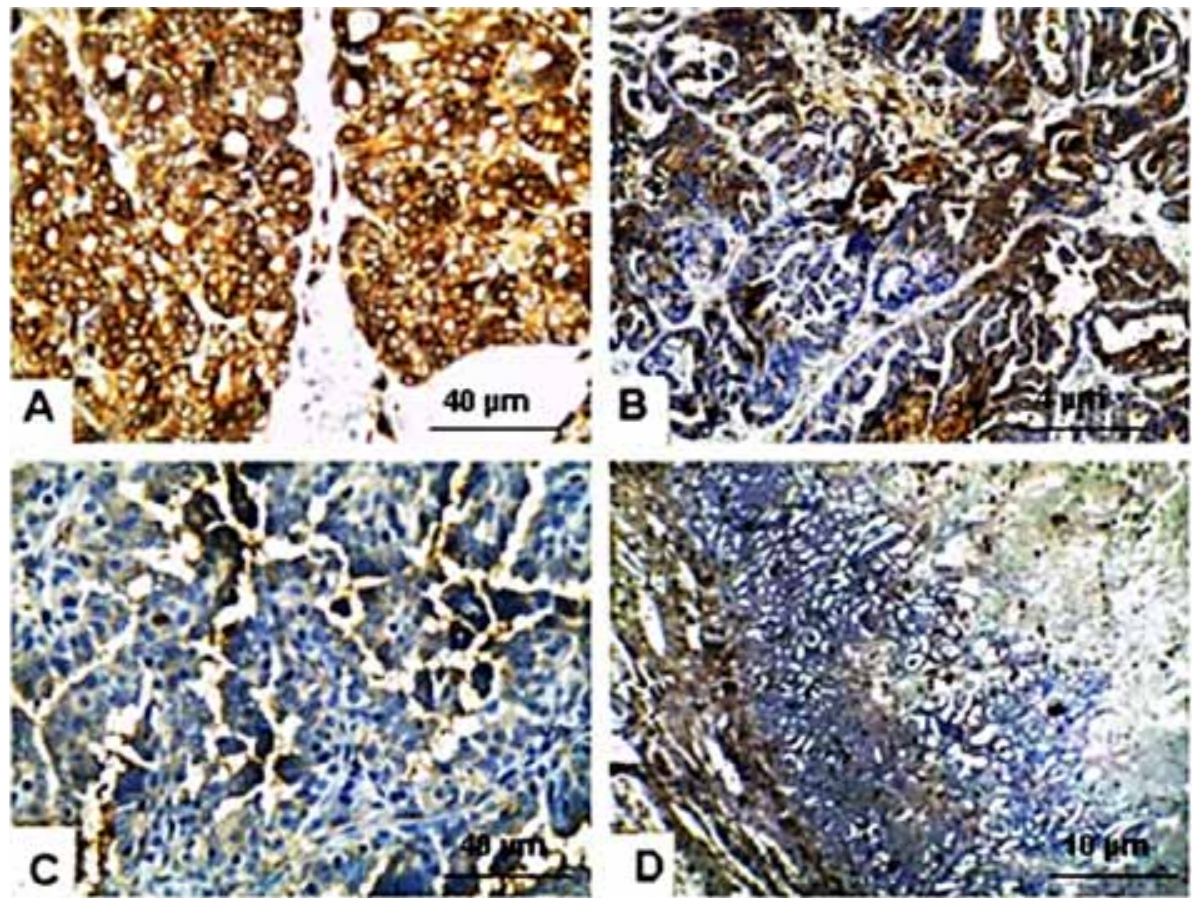

Fig. 1. Her-2 expression in feline mammary lesion samples 